\title{
EXAMPLES OF COMPACT LOCALLY AFFINE SPACES
}

\author{
BY JOHN SCHEUNEMAN
}

Communicated by L. Auslander, February 26, 1971

\begin{abstract}
A construction is given which exhibits many finitelygenerated torsion-free nilpotent groups as fundamental groups of compact complete locally affine spaces.
\end{abstract}

1. Introduction. It is known (see [1] and [2], for example) that there is a close relationship between homogeneous spaces of solvable Lie groups (solvmanifolds) and complete locally affine spaces. One question arising in this connection is what discrete subgroups of solvable (in particular, nilpotent) Lie groups occur as fundamental groups of compact complete locally affine spaces. Of course, finitelygenerated torsion-free abelian groups are such fundamental groups. So are finitely-generated torsion-free two-step nilpotent groups. Aside from this and examples of low dimension, not much seems to be known about fundamental groups of locally affine spaces.

In $\$ 2$, we give a construction which exhibits a wide variety of finitely-generated torsion-free nilpotent groups (all of which are fundamental groups of compact nilmanifolds-see [5]) as fundamental groups of compact complete locally affine spaces. The construction suggests a problem about Lie algebras which we mention in $\S 3$.

2. Construction of examples. Let $N$ be a nilpotent real Lie algebra possessing a nonsingular derivation $D$. Choose a basis $\left\{x_{1}, \cdots, x_{n}\right\}$ of $N$ and identify $N$ with $R^{n}$ via

$$
x_{i} \mapsto\left(\begin{array}{c}
0 \\
: \\
0 \\
0 \\
1 \\
0 \\
\vdots \\
. \\
0
\end{array}\right)
$$

where the 1 is in the $i$ th row. Then the map

AMS 1969 subject classifications. Primary 2250.

Key words and phrases. Solvmanifold, locally affine space, fundamental group. Copyright @ 1971, American Mathematical Society 


$$
E: x \mapsto\left(\begin{array}{cc}
\operatorname{ad}_{x} & D x \\
0 & 0
\end{array}\right)
$$

is a faithful representation of $N$, since

$$
\begin{aligned}
{\left[\left(\begin{array}{cc}
\operatorname{ad}_{x} & D x \\
0 & 0
\end{array}\right),\left(\begin{array}{cc}
\operatorname{ad}_{y} & D y \\
0 & 0
\end{array}\right)\right] } & \\
& =\left(\begin{array}{cc}
\operatorname{ad}_{x} \operatorname{ad}_{y}-\operatorname{ad}_{y} \operatorname{ad}_{x} & {[x, D y]-[y, D x]} \\
0 & 0
\end{array}\right) \\
& =\left(\begin{array}{cc}
\operatorname{ad}_{[x, y]} & D[x y] \\
0 & 0
\end{array}\right)
\end{aligned}
$$

and $D$ is nonsingular.

Now assume the following:

(i) The structure constants of the basis $\left\{x_{1}, \cdots, x_{n}\right\}$ are rational.

(ii) $\left\{x_{1}, \cdots, x_{r_{1}}\right\}$ is a basis for $[N, N],\left\{x_{1}, \cdots, x_{r_{2}}\right\}$ is a basis for $[N[N, N]]$, etc., where $r_{k}<r_{k-1}<\cdots<r_{2}<r_{1}<n$. (In other words, $x$ 's with low subscripts are more nearly central than those with high subscripts.)

(iii) The derivation $D$ is diagonal with respect to $\left\{x_{1}, \cdots, x_{n}\right\}$; i.e., $D x_{i}=\lambda_{i} x_{i}, \lambda_{i} \neq 0$.

REMARK. The Lie algebra $N_{k}$ of $k \times k$ upper triangular nilpotent matrices, together with the basis of matrix units $E_{i, i+r}$ and the derivation $D$ defined by $D E_{i, i+r}=r \lambda E_{i, i+r}$, satisfies all the above hypotheses. There are lots of other examples.

The matrices $E\left(x_{i}\right)$ (computed with respect to the above basis) have the shape

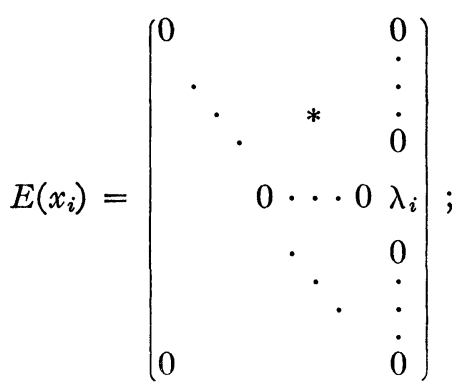

all rows below the $i$ th are zero. Now choose an integer $p$ large enough to make the group $\Gamma$ generated by the upper triangular unipotent matrices $C_{i}=\exp \left(p E\left(x_{i}\right)\right)$ be precisely the set of all $C^{m}=C_{1_{1}^{1}}^{m_{1}} C_{2^{2}}^{m_{2}} \ldots$. $C_{n}^{m_{n}}$, each $m_{i} \in \boldsymbol{Z}$. 
Finally, consider the group $\Gamma$ as a group of affine motions of $R^{n}$, and examine the generic point

$$
\begin{gathered}
C^{\mathbf{m}}\left(\begin{array}{l}
x_{1} \\
\vdots \\
x_{n} \\
1
\end{array}\right) \text { of the } \Gamma \text {-orbit of }\left(\begin{array}{c}
x_{1} \\
\vdots \\
x_{n} \\
1
\end{array}\right) . \\
C^{\mathbf{m}}\left(\begin{array}{l}
x_{1} \\
\vdots \\
x_{n} \\
1
\end{array}\right)=C_{1}^{m_{1}} \cdots C_{n}^{m_{n}}\left(\begin{array}{l}
x_{1} \\
\vdots \\
x_{n} \\
1
\end{array}\right)=\left(\begin{array}{c}
x_{1}+p m_{1} \lambda_{1}+f_{1} \\
\vdots \\
x_{i}+p m_{i} \lambda_{i}+f_{i} \\
\vdots \\
x_{n}+p m_{n} \lambda_{n}+f_{n} \\
1
\end{array}\right) .
\end{gathered}
$$

In view of the shape of the $C_{i}$ 's, $f_{n}=0$, and each $f_{i}$ depends only on the $x$ 's, $m$ 's, and $\lambda$ 's whose subscripts are greater than $i$, and not on the $x$ 's, $m$ 's, and $\lambda$ 's whose subscripts are less than $i$. Therefore, working backward from $n$ to 1 , a unique $\boldsymbol{m}$ can be chosen so that

$$
C^{\mathbf{m}}\left(\begin{array}{l}
x_{1} \\
\vdots \\
x_{n} \\
1
\end{array}\right) \in U_{1} \times \cdots \times U_{n}, \quad \text { where } U_{i} \text { is the interval }\left[0,\left|p \lambda_{i}\right|\right)
$$

in $R$. We then see that $\Gamma$ acts freely on $R^{n}$ and $\Gamma \backslash R^{n}$ is compact, so that $\Gamma$ is the fundamental group of the compact complete locally affine manifold $\Gamma \backslash R^{n}$.

3. A problem on Lie algebras. The construction in $\$ 2$ uses a particular sort of faithful representation of an $n$-dimensional Lie algebra in the Lie algebra of the group of affine motions of $n$-space. If a Lie algebra has a nonsingular derivation, it must be a nilpotent Lie algebra [4], and not every nilpotent Lie algebra has such a derivation [3]. Hence the construction of $\$ 2$ is limited.

We are led to a generalization by analyzing faithful representations

$$
E: x \mapsto\left(\begin{array}{cc}
R_{x} & T x \\
0 & 0
\end{array}\right)
$$

of a Lie algebra $N$, where $T$ is nonsingular. If $E$ is a homomorphism of $N, R$ must be an $n$-dimensional representation (generalizing the 
adjoint representation in $\$ 2$ ), and $T$ must be a nonsingular 1-cocycle of the algebra $N$ with values in the $N$-module $R^{n}$ determined by the representation $R$ (generalizing the derivation $D$ in $\$ 2$ ).

It seems conceivable that every nilpotent Lie algebra may admit a representation $E$ as above. Algebras admitting such representations need not be nilpotent, as shown by the (solvable) example of all real matrices

$$
\left(\begin{array}{llll}
x & 0 & 0 & z \\
0 & x & 0 & y \\
0 & 0 & 0 & x \\
0 & 0 & 0 & 0
\end{array}\right) .
$$

\section{REFERENCES}

1. L. Auslander, On the theory of solvmanifolds and generalization with applications to differential geometry, Proc. Sympos. Pure Math., vol. 3, Amer. Math. Soc., Providence, R.I., 1961, pp. 138-143. MR 23 \#A2483.

2. - - Structure of complete locally affine manifolds, Topology 3 (1964), 131139. MR 28 \#463.

3. J. Dixmier and W. G. Lister, Derivations of nilpotent Lie algebras, Proc. Amer. Math. Soc. 8 (1957), 155-158. MR 18, 659.

4. N. Jacobson, $A$ note on automorphisms and derivations of Lie algebras, Proc. Amer. Math. Soc. 6 (1955), 281-283. MR 16, 897.

5. A. Mal'cev, On a class of homogeneous spaces, Izv. Akad. Nauk SSSR Ser. Mat. 13 (1949), 9-32; English transl., Amer. Math. Soc. Transl. (1) 9 (1962), 276-307. MR $10,507$.

Indiana University, BLOOMINGTon, Indiana 47401 